\title{
Parametrically driven Kerr cavity solitons
}

\author{
Nicolas Englebert, ${ }^{1, *}$ Francesco De Lucia, ${ }^{1,2}$ Pedro Parra-Rivas, ${ }^{1}$ Carlos \\ Mas Arabí, ${ }^{1}$ Pier-John Sazio, ${ }^{2}$ Simon-Pierre Gorza, ${ }^{1}$ and François Leo ${ }^{1}$ \\ ${ }^{1}$ Service OPERA-photonique, Université libre de Bruxelles (U.L.B.), \\ 50 Avenue F. D. Roosevelt, CP 194/5, B-1050 Brussels, Belgium \\ ${ }^{2}$ Optoelectronics Research Centre, University of Southampton, SO17 1BJ, United Kingdom
}

\begin{abstract}
Temporal cavity solitons are optical pulses that propagate indefinitely in nonlinear resonators 1 3. They are currently attracting a lot of attention, both for their many potential applications and for their connection to other fields of science. Cavity solitons are phase locked to a driving laser. This is what distinguishes them from laser dissipative solitons 4 and the main reason why they are excellent candidates for precision applications such as optical atomic clocks [5]. To date, the focus has been on driving Kerr solitons close to their carrier frequency, in which case a single stable localised solution exists for fixed parameters [1. Here we experimentally demonstrate, for the first time, Kerr cavity solitons excitation around twice their carrier frequency. In that configuration, called parametric driving, two solitons of opposite phase may coexist [6]. We use a fibre resonator that incorporates a quadratically nonlinear section and excite stable solitons by scanning the driving frequency. Our experimental results are in excellent agreement with a seminal amplitude equation [7, highlighting connections to hydrodynamic [8, 9] and mechanical systems [10, amongst others [11]. Furthermore, we experimentally confirm that two different phase-locked solitons may be simultaneously excited and harness this multiplicity to generate a string of random bits, thereby extending the pool of applications of Kerr resonators to random number generators [12] and Ising machines [13].
\end{abstract}

The spontaneous formation of patterns is encountered across many fields of science. Spatially extended nonlinear systems may be brought away from equilibrium, where spatiotemporal patterns emerge [14. Examples include convection rolls in heated fluids 15 , vegetation patches in arid regions [16, as well as localised structures in vibrated layers of sand 17 . These complex patterns can often be described by relatively simple reaction/diffusion equations that capture most of the nonlinear dynamics [14]. These so called amplitude equations have been shown to be universal. Very different systems in terms of microscopical physical laws can, under some conditions, be governed by the same macroscopic equation, providing important connections between distinct fields of science.

One such class of equations are the dissipative nonlinear Schrödinger equations (NLSE) which describe pattern formation in charge density condensates, driven plasmas, surface waves and optical resonators amongst others (see [11, 18, and references therein). The conservative NLSE admits exact solitary wave solutions [19. and similar localised structures can be found when dissipation and forcing are added to the system. As in onedimensional oscillators such as the driven pendulum, the forcing can be external [18 or parametric [1]. In the former, the energy is transferred by exciting the systems close to its natural frequency. In the latter, the energy is injected by periodically varying a parameter of the system at twice the system's response frequency. Parametric forcing of spatially extended systems has been intensely studied since the first reports, by Michael Faraday, of pat-

*Electronic address: nicolas.englebert@ulb.ac.be terns on a vibrating surface 20. Parametrically driven NLSE solitons have been reported in hydrodynamics 8 and in chains of oscillators [10, and have been predicted to exist in optical resonators in 1995 6]. They constitute a subclass of optical dissipative solitons 4 along with temporal cavity solitons of the externally forced NLSE [1] 3], which have been shown to underpin the formation of ultra-coherent optical frequency combs 21, 22. Note that temporal cavity solitons are commonly referred to as dissipative Kerr solitons in the context of microresonators 23.

The main differences between externally forced cavity solitons (CSs) and parametrically driven cavity solitons (PCSs) are illustrated in Fig. 1. CSs are solutions of the well known externally driven NLSE (1), often called the Lugiato-Lefever equation [24, 25]. They sit on a homogeneous background and a single phase locked solution exists for fixed detuning and driving power [1, 26]. PCSs, on the other hand, are solutions of the parametrically driven NLSE (2). They lack a homogeneous background and two stable solutions, of opposite phase, may coexist $[6,9$. This multiplicity opens up several new avenues for soliton coalescence [27, 28, as already demonstrated in hydrodynamics 8. Moreover, stable optical pulses of opposite phases can be used to implement random number generators 12 and Ising machines 13. Here, we report the first experimental characterisation of the parametric Kerr cavity soliton. We implement an allfibre singly resonant degenerate optical parametric oscillator (OPO), which is well described by the parametric nonlinear Schrödinger equation (PNLSE). We measure backgroundless sech-shaped optical waves and show that solutions with different phases may coexist in the resonator. As a proof of principle experiment, we generate a short series of random numbers using PCSs. 


\section{a External driving}

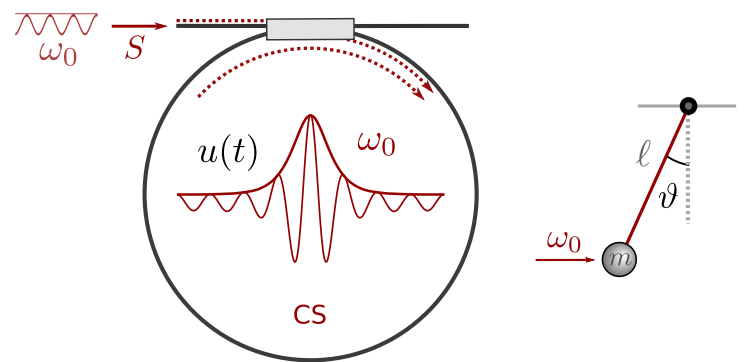

$$
\frac{\partial u}{\partial T}=\left(-1+i\left(|u|^{2}-\Delta\right)+i \frac{\partial^{2}}{\partial \tau^{2}}\right) u+S
$$

\section{b Parametric driving}

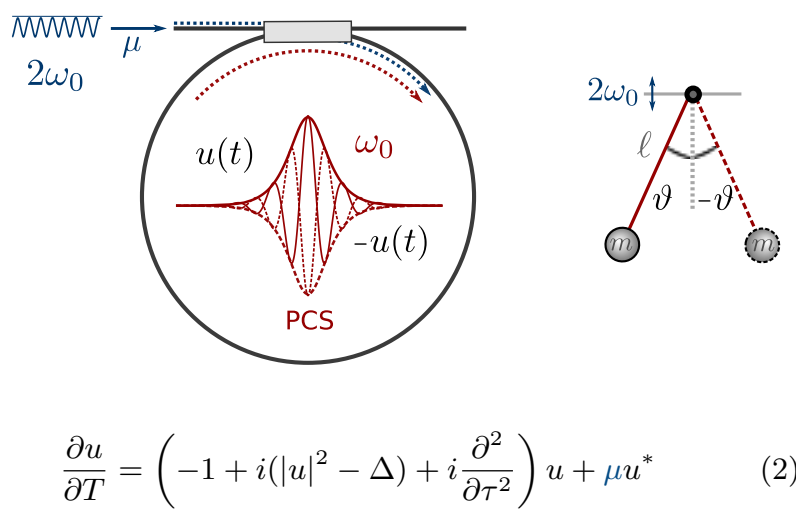

FIG. 1: Illustration of the differences between external and parametric driving. a, Schematic of an optical Kerr resonator pumped close to its natural response frequency. Solitons are solutions of externally driven nonlinear Schrödinger equation (1). Akin to a externally driven damped pendulum, a single phase-locked solution exists. b, Schematic of an optical Kerr resonator pumped close to twice its natural response frequency. Solitons are solutions of the parametrically driven nonlinear Schrödinger equation (2). Akin to a parametrically driven damped pendulum, two different phase-locked solutions exist.
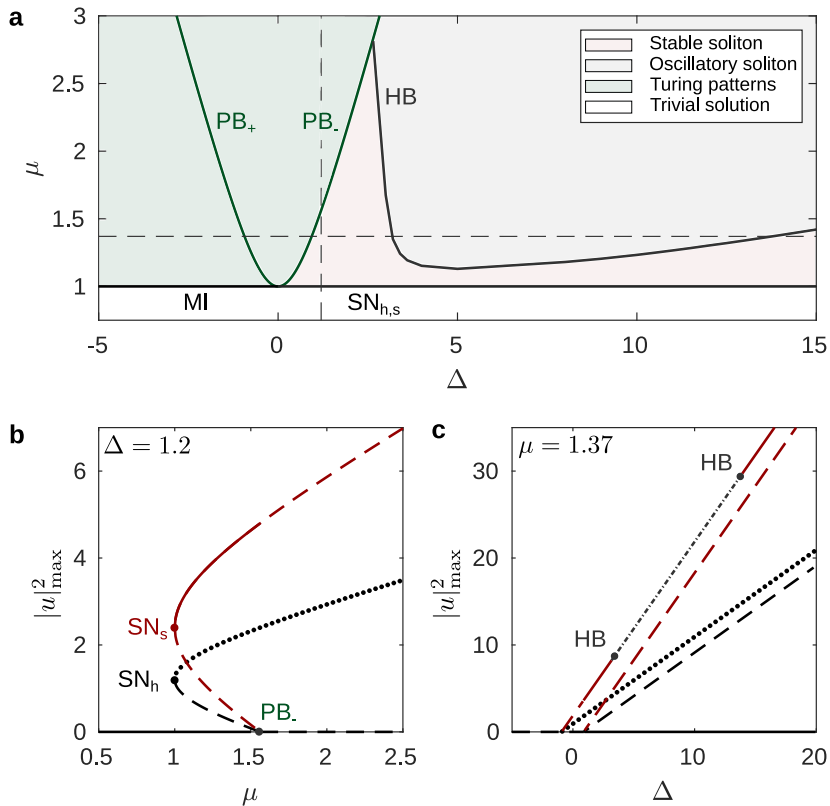

FIG. 2: Bifurcation structure of the PNLSE.

a, Phase diagram in the $(\Delta, \mu)$-parameter space showing the main dynamical regions of the system. The bifurcation lines are the pitchfork bifurcations $\left(\mathrm{PB}_{ \pm}\right)$ corresponding to the degenerate OPO threshold (green solid line) and the Hopf bifurcation (HB) line. The black line at $\mu=1$ corresponds to the saddle-node bifurcation of both the non-trivial homogeneous state $\mathrm{SN}_{h}$ and the soliton state $\mathrm{SN}_{s}$ for $\Delta>0$, and to modulation instability for $\Delta<0$. $\mathbf{b}$, Bifurcation diagram showing the soliton branches (red line) as well as the homogeneous states (black line) as a function of $\mu$ for $\Delta=1.2$. The solid lines corresponds to stable states, dashed lines to homogeneously unstable states and the dotted line to modulationnaly unstable states. c, Bifurcation diagram as a function of $\Delta$ for $\mu=1.37$. The soliton develops breathing behaviour in-between the HBs (see dotted-dashed line).
OPOs are staples of nonlinear optics but the bulk of their usage has been in the homogeneous regime for frequency translation 29. Recently, in the context of frequency comb formation, there has been interest in pattern formation in continuous wave pumped OPOs through cascaded three wave mixing 30, 31. Our work extends the applications of OPOs by showing that they may host phase-locked Kerr solitons.

\section{Bifurcation analysis}

We start by theoretically examining the dynamics of soliton formation in degenerate OPOs incorporating a Kerr section 6]. We consider the dimensionless PNLSE 22. The derivation of the equation and its normalization are detailed in the Supplementary Information. In this equation, there are only two independent parameters: the phase detuning $\Delta$ and pump amplitude $\mu$. They determine the 2-dimensional parameter space, plotted in Fig. 2a, where we show the different nonlinear attractors of the system. The degenerate OPO threshold is located at $\mu=\sqrt{1+\Delta^{2}}$ and correspond to a pitchfork bifurcation (PB) of the trivial state. For negative detunings, that bifurcation is supercritical and the trivial state is modulationally unstable beyond $\mu=1$ [30. The patterns emerging beyond this instability correspond to non degenerate oscillations, which hence has a lower threshold than degenerate emission in that region. For positive detunings, the trivial solution is stable up to $\mu=\sqrt{1+\Delta^{2}}$ and the pitchfork bifurcation is subcritical. An unstable homogeneous state emerges from the trivial solution and folds at the saddle node bifurcation $\mathrm{SN}_{h}$ located at $\mu=1$ (see Fig. 2b). Beyond the fold, the upper branch is modulationally unstable, creating a region where a trivial solution and a modulated pattern coexist. In 


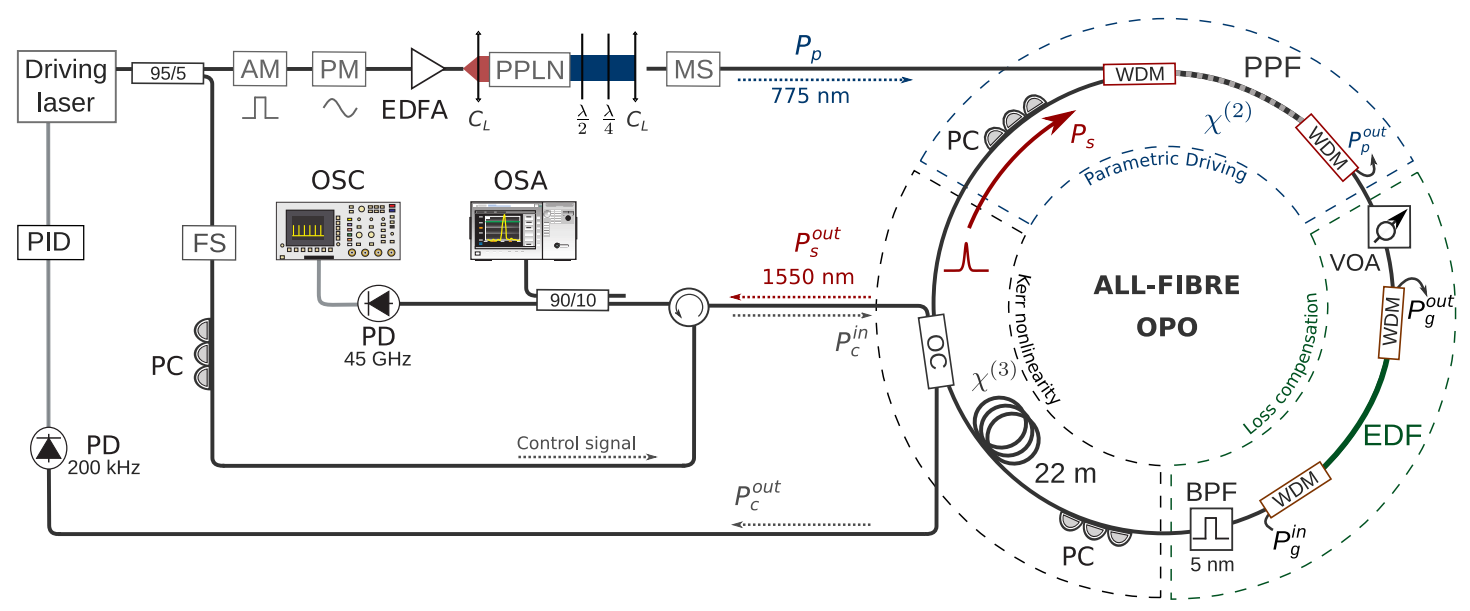

FIG. 3: Experimental set-up. Temporal solitons are excited in an all-fibre degenerate optical parametric oscillator (OPO). It includes a periodically poled fibre (PPF) to provide the parametric gain, a short erbium doped fibre (EDF) pumped by a $1480 \mathrm{~nm}$ laser $\left(P_{g}^{i n}\right)$ for loss compensation and standard single-mode fibre. The cavity is driven by a $775 \mathrm{~nm}$ pump $\left(P_{p}\right)$, obtained by doubling the driving laser frequency in a free-space periodically poled lithium niobate (PPLN). Prior to its conversion, the driving laser is amplitude modulated (AM) and amplified through an erbium doped fibre amplifier (EDFA). A lens $\left(C_{L}\right)$ is used to couple the light to the fibre. Two phase plates and a mode scrambler (MS) are used to limit polarization and modal losses. An output coupler (OC) is included for soliton analysis, using an optical spectrum analyzer (OSA), a fast photodiode (PD) and an oscilloscope (OSC). The cavity is actively stabilised using a proportional-integral-derivative (PID) controller and a counter-propagating beam, frequency shifted (FS) from the driving laser. PC: polarization controller; BPF: bandpass filter; WDM: wavelength division multiplexers; VOA: variable optical attenuator; PM: phase modulator.

that region $(\mu>1)$, the PNLSE admits exact solitary waves of the form $u=\sqrt{2} \beta \operatorname{sech}(\beta t) \exp (i \phi)$ where $\cos (2 \phi)=\mu^{-1}$ and $\beta^{2}=\Delta+\mu \sin (2 \phi)$ [6, 9, 11, 32]. There are two solitons of different amplitude and each can have one of two opposite phases. Both branches, defined as the soliton peak power, are shown in Fig. $2 \mathrm{~b}$ as a function of the driving power. They connect at the saddle node bifurcation $\mathrm{SN}_{s}(\mu=1)$. The solutions corresponding to $\sin (2 \phi)>0$ are always unstable. These soliton branches are remininiscent of the ones describing CSs [33. Conversely, when plotted as a function of the detuning, see Fig. 2 k, both the homogeneous and soliton branches significantly differ from those of CSs [34. Unlike tilted resonances, the stable and saddle PCSs do not connect making the branches infinitely long. In practice, they will be limited by higher order effects (see Supplementary Information). Along the main soliton branch, there are a couple of Hopf bifurcations (HB). Between these bifurcations, the PCSs are unstable and localised oscillatory behaviour as well as complex spatiotemporal dynamics can be found 11. In what follows, we focus on the region where stable soliton formation is predicted.

\section{Experimental setup}

For our experimental investigation of the PCS, we introduce an all-fibre degenerate OPO (see Fig. 3), specifically designed so as to be governed by the PNLSE. It is composed of three main sections made of different fibres. A $27 \mathrm{~cm}$ long periodically poled fibre (PPF) 35, a standard single mode fibre $(21 \mathrm{~m})$, and $52 \mathrm{~cm}$ of erbium doped fibre (EDF). The first two fibres provide, separately, the quadratic and cubic nonlinearities while the EDF is used to compensate the intracavity loss [36]. The OPO is synchronously pumped with highly coherent $650 \mathrm{ps}$ long, flat top, pulses at $775 \mathrm{~nm}$. We use short pulses to keep the gain saturation low. In the region where solitons exist, our system mimicks a high finesse resonator [36. The EDF is pumped with $2 \mathrm{~W}$ at $1480 \mathrm{~nm}$. The corresponding single pass gain is $35 \%$, leading to an effective finesse of 122 around $1550 \mathrm{~nm}$. The $775 \mathrm{~nm}$ driving signal is generated by frequency doubling a highly coherent $1550 \mathrm{~nm}$ laser. It is sent in the cavity through a WDM and removed after the PPF. This single pass configurations ensures that the temporal profile at the driving frequency remains nearly constant, which is crucial when aiming to observe solutions of the PNLSE (see Supplementary Information).

\section{Characterization of the PCS}

In a first experiment, we set the driving power to $10 \mathrm{~W}$ (peak), corresponding to $\mu=1.37$, and scan the laser frequency $(230 \mathrm{kHz} / \mathrm{ms})$. Our results are shown in Fig. 4 . The signal resonance, measured around $1550 \mathrm{~nm}$, is reminiscent of that observed in externally pumped Kerr resonators [3]. The signal average power gradually increases until it reaches the bistable region where it suddenly drops, indicating the formation of localised structures. The small plateau emerging at that point corresponds to the soliton branch shown in Fig. 22. In the context of externally driven Kerr resonators, it is often called the 

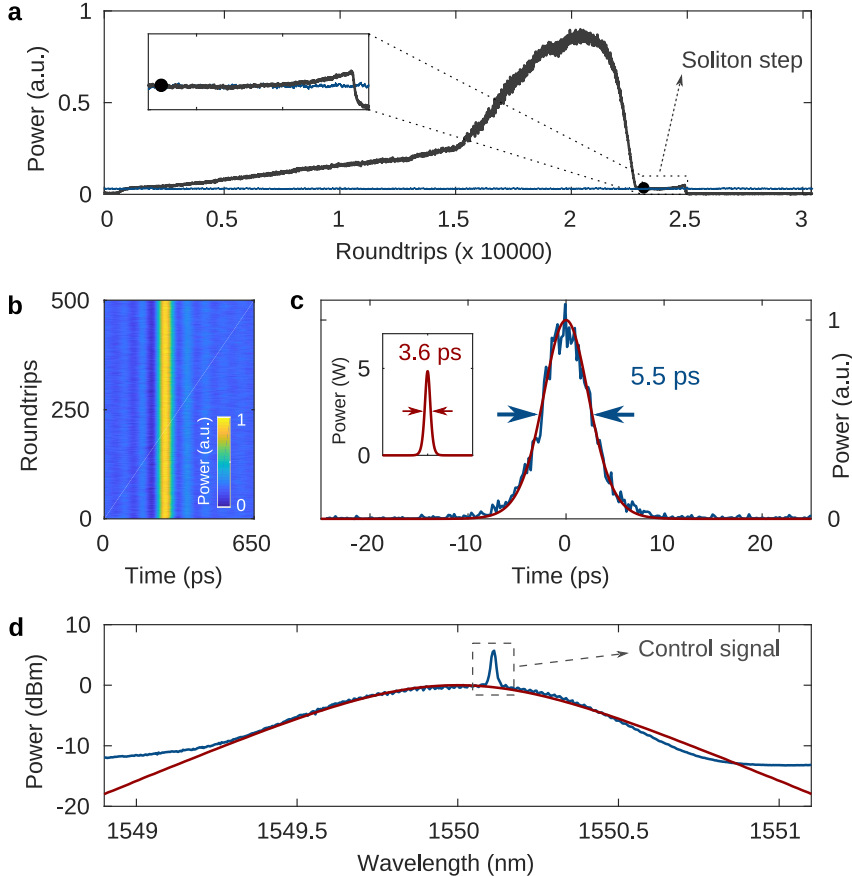

FIG. 4: Characterisation of the Parametric

Cavity Soliton. a, Forward scan (black line) through a resonance for $P_{p}=10 \mathrm{~W}$. The dot highlights the stabilisation setpoint $(\Delta=1.2)$. The blue line corresponds to the output power when the cavity is actively stabilised around that level. b, Oscilloscope recording - taken several seconds after the excitation process - showing a stable, resolution limited, pulse exiting the cavity. c, Experimental (blue line) and theoretical (red line) autocorrelations traces. The inset shows the theoretical profile of the corresponding background-free soliton. d, Experimental (blue line) and theoretical (red line) spectra at the cavity output. The narrow peak corresponds to back reflections of the control signal.

soliton step as pulses tend to merge one by one, leading to a stairs-shaped transmission curve [3]. Additional higher resolution measurements of the nonlinear transmission of the cavity, including multi soliton steps, are shown in the Supplementary Information. We readily note an important difference between our experimental scans and the analytical branch shown in Fig. 2. The soliton step in our experiments has a finite extension while the theoretical branch grows indefinitely with increasing $\Delta$. First, we stress that frequency scans are inherently dynamical such that the measured output power is not necessarily representative of steady state solutions at the corresponding detuning. Second, higher order effects limit the branch in optical parametric oscillators (see Supplementary Information). In our experiment, however, the soliton collapse is due to the 5-nm, flat top, intracavity filter we use to prevent lasing at shorter wavelengths [36]. As the detuning is ramped up, so is the soliton's spectral width, such that the filter eventually prevents stable soliton formation.

Next, we use a control signal to stabilise the system in the soliton region (see Methods). The average output power when the detuning is set to $\Delta=1.2\left(\delta_{0}=0.03\right)$ is shown in Fig. 4a. A high-resolution (80 ps) recording of the corresponding cavity output is shown in Fig. $4 \mathrm{~b}$. A resolution limited pulse can be seen exiting the cavity every rountrip time. Further temporal (Fig. 4c) and spectral (Fig. 4 $\mathrm{d}$ ) characterisations confirm that a short (3.6 ps) pulse is circulating in the cavity. The agreement with the analytic soliton solution of the PNLSE is excellent. The experimental spectral background corresponds to the ASE emitted by the intracavity amplifier [36]. These measurements confirm that our novel system is governed by the PNLSE in that region and constitute, to the best of our knowledge, the first experimental observation of its well known soliton in optics.

\section{Random bits generation}

Parametrically driven Kerr cavity solitons are phase locked to a driving laser, as are externally driven CSs which attract a lot of attention because of their inherent stability. The additional advantage of the PCS is its multiplicity. Owing to the $\mathbb{Z}_{2}$-symmetry of the PNLSE, two attractors, which have the same amplitude but opposite phase, may coexist in the cavity, adding a degree of freedom to Kerr resonators. In particular, it opens the possibility to use Kerr solitons in applications, such as random bit generators [12] and Ising machines 13, which require two different attractors. To confirm this potential, we design a proof of principle experiment of random number generation. The concept is simple. When a soliton is spontaneously excited, it has a $50 \%$ chance of locking to the pump with one of the two possible phase relations. By exciting multiple solitons, and extracting the phase, we can generate a random sequence of bits. For this demonstration, we phase modulate the pump beam so as to excite a series of equally spaced single solitons. The physics behind soliton attraction to phase maxima is similar to that of CSs [37] and is detailed in the Supplementary Information. A low modulation frequency $(4.6 \mathrm{GHz})$ is chosen to be able to resolve individual solitons on the oscilloscope. We extract a portion of the $1550 \mathrm{~nm}$ driving laser, prior to its frequency doubling, and use it as a local oscillator for coherent detection (see Fig. 5a). We excite two solitons in the cavity and send both the reference and the combined beams to a fast photodetector. The results are shown in Fig. 5b-c. As expected, the reference, corresponding to the intensity, displays identical traces separated by 220 ps. After interfering with the local oscillator however, two different amplitudes are measured. These measurements confirm that solitons of different phases are excited in the cavity. In a second series of experiments, we expand the pulse width to host four solitons and perform three distinct resonance scans. Our results are shown in Fig. 5 d-f. By assigning a binary value to each soliton, our results correspond to a series 
of 4-bits random numbers, highlighting the potential of PCSs for applications. Moreover, our measurements confirm that the solitons are phase-locked, as only 2 distinct amplitudes are measured across the different scans.

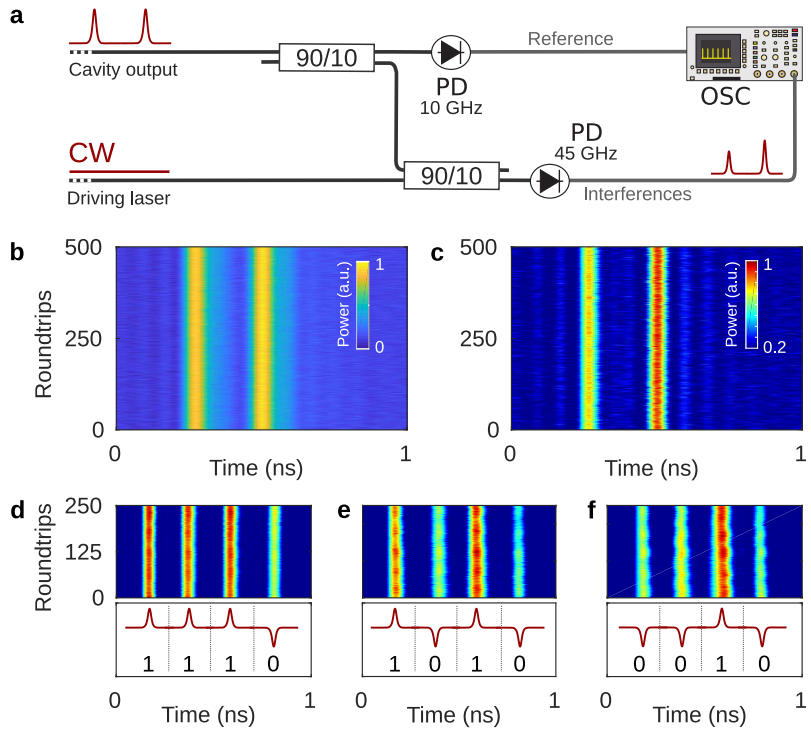

FIG. 5: Random bits generation. a, Experimental set-up for coherent detection. $\mathbf{b}$, Direct detection of two PCSs. c, Coherent detection of two PCSs, highlighting the two different phases. d-e, Sequences of four random bits generated through PCS formation.

\section{Discussion}

In summary, we investigated Kerr soliton formation in singly resonant optical parametric oscillators. We built a novel system that is well described by the seminal parametric nonlinear Schrödinger equation when driven with a frequency close to twice that of a longitudinal mode. We theoretically showed that a couple of stable solitons exist in a broad region of experimental parameters. Our measurements confirm the existence of a backgroundless, sech-shaped and phase locked optical pulse in that region. Its temporal and spectral profiles are in excellent agreement with the soliton solution of the PNLSE. The same profile corresponds to the well known non propagating hydrodynamic soliton [8, 9. Here, the soliton propagates along the resonator and forms an ultra-stable pulse train at the output. The phase-locking ensures minimal jitter and the output spectrum consists in an ultra-coherent frequency comb. Importantly, the large central peak inherent to external driving is absent. Moreover, we showed that applications of PCSs go beyond frequency comb generation. The two different phases can be leveraged for random number generation, as demonstrated above, or physical Ising machines. The latter has already been implemented using a synchronously pumped degenerate OPO 13, but the number of individual spins is limited by the repetition rate of the pump laser. Our results show that a grid of individual spins, as dense the input phase modulation, can be generated in a long fibre cavity. Be- cause the number of potential connections scales as $N^{2}$, a $40 \mathrm{GHz}$ phase modulation would lead to a 3 orders of magnitude increase in the number of spin-spin couplings as compared to the state of the art [13.

\section{Methods}

\section{Linear stability analysis}

The temporal linear stability of the steady-state solutions, shown in Fig. 2, has been computed by solving the eigenvalue problem $\mathcal{L} \psi=\sigma \psi$, obtained from the linearization of Eq. (2) around a given steady state, where $\mathcal{L}$ the linear operator evaluated at such state, and $\sigma$ and $\psi$ are, respectively, the eigenvalues and eigenfunctions of $\mathcal{L}$. This problem can be easily solved analytically for the homogeneous $u_{h}$ state as shown in the Supplementary Information. For the soliton state stability, we have adopted a numerical approach. We compute the eigenvalues of the Jacobian matrix obtained from $\mathcal{L}$ after spatial discretization in a $N=1024$ points grid.

\section{Experimental set-up}

The all-fibre optical parametric oscillator (OPO) is made of a section $\left(L_{1}=27 \mathrm{~cm}\right)$ of periodically poled silica fibre $(\mathrm{PPF})$, a section $\left(L_{2}=21 \mathrm{~m}\right)$ of standard telecommunication singlemode silica fibre $(\mathrm{SMF}-28)$ and a section $\left(L_{3}=52 \mathrm{~cm}\right)$ of erbium doped fibre (EDF). The PPF has a second-order nonlinear parameter of $\kappa=0.04 \mathrm{~W}^{-1 / 2} \mathrm{~m}^{-1}$ and a phase-matching wavelength of $1548.8 \mathrm{~nm}$ at room temperature. This wavelength is increased up to $1549.72 \mathrm{~nm}$ to be in the tuning range of the driving laser by placing the fibre in a stabilised oven at $36^{\circ} \mathrm{C}$. Two wavelength division multiplexers (WDMs) are used to combine the $775 \mathrm{~nm}$ pump with the intracavity signal, and to reject the remaining pump power at the fibre output. Two different polarization controllers are used. One to align the pump polarization with the phase-matched eigenmode of the PPF and the other to align the signal polarization with one of the two eigenmodes of the cavity. The EDF (Liekki ${ }^{\circledR}$ ER16-8/125) provides the optical gain. Two wavelength division multiplexers (WDMs) are inserted in the cavity to combine the $1480 \mathrm{~nm}$ pump with the intracavity signal, and to reject the unabsorbed power at the amplifier output. Its length is empirically set so that the gain is slightly larger than the intrinsic cavity loss. We then use a variable optical attenuator to increase the loss and ensure the cavity is below the lasing threshold. An optical bandpass filter ( $5 \mathrm{~nm}$ at $0.5 \mathrm{~dB}$, centred on $1550 \mathrm{~nm}$ ) hinders laser emission at shorter wavelengths. The cavity contains a $99 / 1$ coupler used either to inject the control signal into the cavity or to extract part of the intracavity power. The total intracavity loss, excluding the doped fibre, is $40 \%$. The driving continuous wave $(\mathrm{CW})$ laser is a Koheras Adjustik ${ }^{\mathrm{TM}}$ E15 with a sub-100 Hz linewidth. Its wavelength is set to $1549.72 \mathrm{~nm}$, on the edge of the tuning range $(1 \mathrm{~nm})$ to coincide with the PPF phase-matching wavelength. The laser output is first modulated with a Mach-Zehnder amplitude modulator (bandwidth: $12 \mathrm{GHz}$, extinction ratio: $30 \mathrm{~dB}$ ), driven by a pattern generator connected to an RF clock. The pulsed beam is then amplified with an erbium doped fibre amplifier (EDFA) and converted to its second harmonic through a $4 \mathrm{~cm}$ long periodically poled lithium niobate (Covesion ${ }^{\circledR}$ MSHG1550-0.5-40, $\kappa=2.5 \mathrm{~W}^{-1 / 2} \mathrm{~m}^{-1}$ ) in a free-space section. Using dichroic mirrors, the unconverted field is attenuated by $125 \mathrm{~dB}$ such that only the pump is injected into the fibre. To minimize 
both polarization and modal losses at the first WDM, a halfand quarter-wave plate (free-space) and a mode scrambler are used. The cavity resonances are measured by scanning the frequency of the driving laser and recording the average power at the output coupler, $P_{s}^{\text {out }}$, with a $200 \mathrm{kHz}$ photodiode (see e.g. Figure 4a). To stabilise the cavity, a control signal is generated by extracting a portion of the driving laser power through a $95 / 5$ coupler and shifting its frequency with a tunable frequency-shifter $(110 \pm 5 \mathrm{MHz})$. Using a circulator and a polarization controller, the counter-propagative control signal, with a power of $P_{c}^{i n}$, is sent to the cavity on the orthogonal polarization eigenmode to avoid seeding the OPO. The cavity detuning is stabilised by slightly changing the driving laser wavelength to maintain a constant control signal output power $P_{c}^{\text {out }}$. The feedback signal is generated by a proportional-integral-derivative (PID) controller (Toptica DigiLock 110), driven by a photodiode. The system is stabilised on the slope of the linear resonance of the control signal. Knowing the cavity birefringence, the detuning of the signal can be extracted. It can then be modified by changing the control signal frequency [38]. Part of the intracavity power is extracted at the output coupler to characterize the solitons. The spectrum of the parametric cavity soliton (PCS) is recorded on an optical spectrum analyser $(0.1 \mathrm{~nm}$ resolution bandwidth). Time measurements are carried out with a fast photodiode (45 GHz bandwidth) and an oscilloscope (10 GHz bandwidth, 10 Gsample.s ${ }^{-1}$ ). The intensity autocorrelation trace is directly acquired at the cavity output. For this measurement, a commercial EDFA is used to increase the average output power to $\sim 70 \mathrm{~mW}$.

\section{PPF fabrication}

The PPF is a $125 \mu \mathrm{m}$ outside diameter cladding fibre with a Germania-doped glass core of $4 \mu \mathrm{m}$ diameter and a numerical aperture $\mathrm{NA}=0.17$. Two $27 \mu \mathrm{m}$ diameter channels run adjacent to the fibre core at a distance of respectively $13.6 \mu \mathrm{m}$ and $7.2 \mu \mathrm{m}$ from the core's edges. The fibre is first thermally poled in single anode configuration at $265^{\circ} \mathrm{C}$ with an electric potential of $+8 \mathrm{kV}$ applied to the embedded electrode, for 2 hours [39]. The second order nonlinearity created via thermal poling is then erased periodically by means of a CW argon ion laser frequency doubled to $244 \mathrm{~nm}$, equipped with an acousto-optic modulator (AOM) used to modulate the laser output. The laser is focused to a circular spot, $20 \mu \mathrm{m}$ in diameter, while the poled fibre is clamped onto a linear stage by two fibre rotator clamps. The laser is modulated using the AOM while translating the fibre core through the spot to achieve a grating of the desired duty cycle and period. For the grating a fluence of $14 \mathrm{~J} / \mathrm{cm}^{2}$ and a duty cycle of $5 \%$ was used to periodically erase the nonlinearity. The period of the grating was chosen to be $55 \mu \mathrm{m}$ in order to have quasi-phase matching at a wavelength around $1550 \mathrm{~nm}$.

\section{PCS excitation and stabilisation}

For all measurements depicted in Fig 4, the cavity is synchronously pumped with 650 ps flat-top pulses whose repetition frequency matches the cavity free-spectral range (FSR). The cavity detuning is stabilised by locking the control signal through-port transmission at $90 \%$ (i.e. $P_{c}^{\text {out }} / P_{c}^{\text {in }}=0.9$ ). Once stabilised, the control signal frequency is increased with the frequency-shifter (FS) until the signal output power drops to the soliton-step power (Fig. 4a, red). This coincides with the emergence of a background-free sech squared-like spectrum, corresponding to the generation of a single PCS. Temporal and spectral measurements are then carried out.

\section{Coherent detection measurement}

To demonstrate the existence of PCS with opposite phases, the cavity is synchronously pumped with $1 \mathrm{~ns}$ or $1.9 \mathrm{~ns}$ flat-top pulses. On these pump pulses, we also imprint a 4.6 $\mathrm{GHz}$ phase modulation (PM) using a phase-modulator. As for CSs 37, 40, PCS are attracted by PM maxima (see Supplementary Information). When scanning the resonance, we generate up to four PCSs, separated by 220 ps. Using a $90 / 10$ coupler, most of the cavity output power $P_{s}^{\text {out }}$ is sent to a $10 \mathrm{GHz}$ photodiode [i.e. reference beam on Fig. 5a]. The remaining power is combined with part of the driving laser power, obtained by bypassing the frequency-shifter, through another $90 / 10$ coupler. The result of the interference is sent to a $45 \mathrm{GHz}$ photodiode for coherent detection.

\section{Acknowledgements}

We are grateful to Michaël Fita Codina for the manufacturing of experimental components and to Pascal Kockaert and Costantino Corbari for fruitfull discussions. This work was supported by funding from the European Research Council (ERC) under the European Union's Horizon 2020 research and innovation programme (grant agreement No 757800). N.E. acknowledges the support of the Fonds pour la formation à la Recherche dans l'Industrie et dans l'Agriculture (FRIA, Belgium). P.P.R. acknowledges the support of the "Fonds de la Recherche Scientifique" (FNRS, Belgium).

\section{Author Contributions}

N.E. designed and performed the experiments, supervised by S-P.G. F.D.L. and P.J.S. manufactured the periodically poled fibre. NE derived and simulated the mean-field model. P.P.R. and C.M.A. performed the bifurcation and linear stability analysis of the mean-field model. F.L. supervised the overall project and wrote the manuscript. All authors discussed the results and contributed to the final manuscript.
[1] Wabnitz, S. Suppression of interactions in a phaselocked soliton optical memory. Optics Letters 18, 601603 (1993). URL https://www.osapublishing.org/ol/ abstract.cfm?uri=ol-18-8-601. Publisher: Optical Society of America.

[2] Leo, F. et al. Temporal cavity solitons in one-dimensional
Kerr media as bits in an all-optical buffer. Nature Photonics 4, 471-476 (2010). URL https://www.nature. com/articles/nphoton.2010.120 Number: 7 Publisher: Nature Publishing Group.

[3] Herr, T. et al. Temporal solitons in optical microresonators. Nature Photonics 8, 145-152 
(2014). URL https://www.nature.com/articles/ nphoton.2013.343 Number: 2 Publisher: Nature Publishing Group.

[4] Grelu, P. \& Akhmediev, N. Dissipative solitons for mode-locked lasers. Nature Photonics 6, 8492 (2012). URL https://www.nature.com/articles/ nphoton.2011.345 Number: 2 Publisher: Nature Publishing Group.

[5] Newman, Z. L. et al. Architecture for the photonic integration of an optical atomic clock. Optica 6, 680-685 (2019). URL https://www . osapublishing.org/optica/ abstract.cfm?uri=optica-6-5-680 Publisher: Optical Society of America.

[6] Longhi, S. Ultrashort-pulse generation in degenerate optical parametric oscillators. Optics Letters 20, 695697 (1995). URL https://www. osapublishing.org/ol/ abstract.cfm?uri=ol-20-7-695. Publisher: Optical Society of America.

[7] Longhi, S. Hydrodynamic equation model for degenerate optical parametric oscillators. Journal of Modern Optics 43, 1089-1094 (1996). URL https://doi.org/10. 1080/09500349608232787. Publisher: Taylor \& Francis _eprint: https://doi.org/10.1080/09500349608232787.

[8] Wu, J., Keolian, R. \& Rudnick, I. Observation of a Nonpropagating Hydrodynamic Soliton. Physical Review Letters 52, 1421-1424 (1984). URL https://link.aps. org/doi/10.1103/PhysRevLett.52.1421. Publisher: American Physical Society.

[9] Miles, J. W. Parametrically excited solitary waves. Journal of Fluid Mechanics 148, 451460 (1984). URL https://www.cambridge.org/ core/journals/journal-of-fluid-mechanics/ article/parametrically-excited-solitary-waves / F6E726F7DD524A2F8F8C9F3EC0CBEAF4\# Publisher: Cambridge University Press.

[10] Denardo, B. et al. Observations of localized structures in nonlinear lattices: Domain walls and kinks. Physical Review Letters 68, 1730-1733 (1992). URL https://link. aps.org/doi/10.1103/PhysRevLett.68.1730 Publisher: American Physical Society.

[11] Bondila, M., Barashenkov, I. V. \& Bogdan, M. M. Topography of attractors of the parametrically driven nonlinear Schrödinger equation. Physica D: Nonlinear Phenomena 87, 314-320 (1995). URL http://www.sciencedirect. com/science/article/pii/0167278995001260

[12] Marandi, A., Leindecker, N. C., Vodopyanov, K. L. \& Byer, R. L. All-optical quantum random bit generation from intrinsically binary phase of parametric oscillators. Optics Express 20, 19322-19330 (2012). URL https://www.osapublishing.org/oe/ abstract.cfm?uri=oe-20-17-19322 . Publisher: Optical Society of America.

[13] Inagaki, T. et al. A coherent Ising machine for 2000-node optimization problems. Science 354, 603-606 (2016). URL https://science.sciencemag.org/content/354/ 6312/603 Publisher: American Association for the Advancement of Science Section: Report.

[14] Cross, M. C. \& Hohenberg, P. C. Pattern formation outside of equilibrium. Reviews of Modern Physics 65, 851-1112 (1993). URL https://link.aps .org/doi/10. 1103/RevModPhys.65.851. Publisher: American Physical Society.

[15] Ahlers, G., Grossmann, S. \& Lohse, D. Heat transfer and large scale dynamics in turbulent rayleigh- bénard convection. Reviews of Modern Physics 81, 503537 (2009). URL https://link.aps .org/doi/10.1103/ RevModPhys.81.503. Publisher: American Physical Society.

[16] Lejeune, O., Tlidi, M. \& Couteron, P. Localized vegetation patches: A self-organized response to resource scarcity. Physical Review E 66, 010901 (2002). URL https://link.aps.org/doi/10.1103/ PhysRevE.66.010901 Publisher: American Physical Society.

[17] Umbanhowar, P. B., Melo, F. \& Swinney, H. L. Localized excitations in a vertically vibrated granular layer. Nature 382, 793-796 (1996). URL https://www.nature.com/ articles/382793a0. Number: 6594 Publisher: Nature Publishing Group.

[18] Barashenkov, I. V. \& Smirnov, Y. S. Existence and stability chart for the ac-driven, damped nonlinear schrodinger solitons. Physical Review E 54, 57075725 (1996). URL https://link.aps.org/doi/10. 1103/PhysRevE.54.5707| Publisher: American Physical Society.

[19] Zakharov, V. E. \& Shabat, A. B. Exact Theory of Two-dimensional Self-focusing and One-dimensional Self-modulation of Waves in Nonlinear Media. Soviet Journal of Experimental and Theoretical Physics 34, 62 (1972). URL http://adsabs.harvard.edu/abs/ 1972JETP...34 ..62Z

[20] Faraday, M. On a peculiar class of acoustical figures; and on certain forms assumed by groups of particles upon vibrating elastic surfaces. Philosophical Transactions of the Royal Society of London 121, 299-340 (1831). URL https://royalsocietypublishing.org/ doi/10.1098/rstl.1831.0018 Publisher: Royal Society.

[21] Coen, S., Randle, H. G., Sylvestre, T. \& Erkintalo, M. Modeling of octave-spanning Kerr frequency combs using a generalized mean-field lugiatolefever model. Optics Letters 38, 37-39 (2013). URL https://www.osapublishing.org/ol/abstract. cfm?uri=ol-38-1-37. Publisher: Optical Society of America.

[22] Parra-Rivas, P., Gomila, D., Matías, M. A., Coen, S. \& Gelens, L. Dynamics of localized and patterned structures in the Lugiato-Lefever equation determine the stability and shape of optical frequency combs. Physical Review A 89, 043813 (2014). URL https://link .aps .org/ doi/10.1103/PhysRevA.89.043813 Publisher: American Physical Society.

[23] Kippenberg, T. J., Gaeta, A. L., Lipson, M. \& Gorodetsky, M. L. Dissipative Kerr solitons in optical microresonators. Science 361 (2018). URL https://science. sciencemag.org/content/361/6402/eaan8083. Publisher: American Association for the Advancement of Science Section: Review.

[24] Lugiato, L. A. \& Lefever, R. Spatial Dissipative Structures in Passive Optical Systems. Physical Review Letters 58, 2209-2211 (1987). URL https://link.aps.org/ doi/10.1103/PhysRevLett.58.2209. Publisher: American Physical Society.

[25] Haelterman, M., Trillo, S. \& Wabnitz, S. Dissipative modulation instability in a nonlinear dispersive ring cavity. Optics Communications 91, 401-407 (1992).

[26] Nozaki, K. \& Bekki, N. Chaotic solitons in a plasma driven by an RF field. Journal of the Physical Society 
of Japan 54, 2363-2366 (1985). URL http://adsabs. harvard.edu/abs/1985JPSJ . . 54.2363N

[27] Wang, Y. et al. Universal mechanism for the binding of temporal cavity solitons. Optica 4, 855-863 (2017). URL https://www .osapublishing.org/optica/ abstract.cfm?uri=optica-4-8-855 . Publisher: Optical Society of America.

[28] Cole, D. C., Lamb, E. S., Del'Haye, P., Diddams, S. A. \& Papp, S. B. Soliton crystals in Kerr resonators. Nature Photonics 11, 671-676 (2017). URL https:// www.nature.com/articles/s41566-017-0009-z Number: 10 Publisher: Nature Publishing Group.

[29] Myers, L. E. et al. Quasi-phase-matched optical parametric oscillators in bulk periodically poled $\mathrm{LiNbO}_{3}$. Journal of the Optical Society of America B 12, 2102-2116 (1995). URL https://www.osapublishing.org/josab/ abstract.cfm?uri=josab-12-11-2102 Publisher: Optical Society of America.

[30] Mosca, S. et al. Modulation Instability Induced Frequency Comb Generation in a Continuously Pumped Optical Parametric Oscillator. Physical Review Letters 121, 093903 (2018). URL https://link.aps.org/doi/ 10.1103/PhysRevLett.121.093903 Publisher: American Physical Society.

[31] Bruch, A. W. et al. Pockels soliton microcomb. $\mathrm{Na}$ ture Photonics 1-7 (2020). URL https://www nature. com/articles/s41566-020-00704-8. Publisher: Nature Publishing Group.

[32] Pérez-Arjona, I., Roldán, E. \& de Valcárcel, G. J. Theory of quantum fluctuations of optical dissipative structures and its application to the squeezing properties of bright cavity solitons. Physical Review A $\mathbf{7 5}$, 063802 (2007). URL https://link.aps.org/doi/10. 1103/PhysRevA.75.063802. Publisher: American Physical Society.

[33] Scroggie, A. J. et al. Pattern formation in a passive Kerr cavity. Chaos, Solitons \& Fractals 4, 1323-1354 (1994). URL http://www.sciencedirect.com/science/ article/pii/0960077994900841

[34] Coen, S. \& Erkintalo, M. Universal scaling laws of Kerr frequency combs. Optics Letters 38, 17901792 (2013). URL https://www.osapublishing.org/ ol/abstract.cfm?uri=ol-38-11-1790 Publisher: Optical Society of America.

[35] De Lucia, F., Keefer, D. W., Corbari, C. \& Sazio, P. J. A. Thermal poling of silica optical fibers using liquid electrodes. Optics Letters 42, 69-72 (2017). URL https://www.osapublishing.org/ol/ abstract.cfm?uri=ol-42-1-69 Publisher: Optical Society of America.

[36] Englebert, N., Gorza, S.-P. \& Leo, F. Temporal Solitons in a Coherently Driven Active Resonator. arXiv:200\%.15630 [physics] (2020). URL http://arxiv. org/abs/2007.15630 ArXiv: 2007.15630.

[37] Jang, J. K., Erkintalo, M., Coen, S. \& Murdoch, S. G. Temporal tweezing of light through the trapping and manipulation of temporal cavity solitons. Nature Communications 6, 1-7 (2015). URL https://www . nature.com/ articles/ncomms 8370

[38] Li, Z. et al. Experimental observations of bright dissipative cavity solitons and their collapsed snaking in a Kerr resonator with normal dispersion driving. Optica 7, 1195-1203 (2020). URL https://www.osapublishing. org/optica/abstract.cfm?uri=optica-7-9-1195
Publisher: Optical Society of America.

[39] De Lucia, F. et al. Single is better than double: theoretical and experimental comparison between two thermal poling configurations of optical fibers. Optics Express 27, 27761-27776 (2019). URL https://www.osapublishing.org/oe/abstract. cfm?uri=oe-27-20-27761. Publisher: Optical Society of America.

[40] Jang, J. K. et al. Controlled merging and annihilation of localised dissipative structures in an AC-driven damped nonlinear Schrödinger system. New Journal of Physics 18, 033034 (2016). URL https://doi.org/10.1088\% 2F1367-2630\%2F18\%2F3\%2F033034. Publisher: IOP Publishing. 


\title{
Parametrically driven Kerr cavity solitons
}

\author{
Nicolas Englebert, ${ }^{1, *}$ Francesco De Lucia, ${ }^{1,2}$ Pedro Parra-Rivas, ${ }^{1}$ Carlos \\ Mas Arabí, ${ }^{1}$ Pier-John Sazio, ${ }^{2}$ Simon-Pierre Gorza, ${ }^{1}$ and François Leo ${ }^{1}$ \\ ${ }^{1}$ Service OPERA-photonique, Université libre de Bruxelles (U.L.B.), \\ 50 Avenue F. D. Roosevelt, CP 194/5, B-1050 Brussels, Belgium \\ ${ }^{2}$ Optoelectronics Research Centre, University of Southampton, SO17 1BJ, United Kingdom
}

\begin{abstract}
This article contains the Supplementary Information for the manuscript entitled "Parametrically driven Kerr cavity solitons". We derive the parametrically driven nonlinear Schrödinger equation and perform a stability analysis of its stationary states. We also theoretically describe how parametric cavity solitons behave when the driving field is phase-modulated. Finally, additional experimental results are given.
\end{abstract}

\section{MEAN-FIELD MODEL}

Pattern formation in our system can be described by a single mean-field equation at the signal frequency $\omega_{0}$. We here detail its derivation starting from the full lumped model. We start with a description of a passive singly resonant optical parametric oscillator incorporating a Kerr section. The cavity boundary conditions for both the signal field $A$ at $\omega_{0}$ and the pump field $B$ at $2 \omega_{0}$ read [1]

$$
\begin{aligned}
& A_{m+1}(0, \tau)=\sqrt{\mathcal{T}} A_{m}(L, \tau) \mathrm{e}^{i \varphi}, \\
& B_{m+1}(0, \tau)=B_{i n},
\end{aligned}
$$

where $m$ is the roundtrip number, $\mathcal{T}=\prod_{k} \mathcal{T}_{k}$ denotes the total insertion loss of all cavity components (polarization controllers, coupler, WDMs, ...) and $\varphi_{0}$ is the linear phase accumulated by the signal $\left(\omega_{0}\right)$ over one roundtrip. The evolution of these fields in the periodically poled fibre (PPF) is given by the two following equations [2]

$$
\begin{array}{r}
\frac{\partial A_{m}}{\partial z}=-\left(\frac{\alpha_{s}^{(1)}}{2}+i \frac{\beta_{2, s}^{(1)}}{2} \frac{\partial^{2}}{\partial \tau^{2}}\right) A_{m}+i \kappa B_{m} A_{m}^{*} \mathrm{e}^{-i \Delta \beta z} \\
\frac{\partial B_{m}}{\partial z}=-\left(\frac{\alpha_{p}^{(1)}}{2}+\Delta \beta_{1}^{(1)} \frac{\partial}{\partial \tau}+i \frac{\beta_{2, p}^{(1)}}{2} \frac{\partial^{2}}{\partial \tau^{2}}\right) A_{m} \\
+i \kappa A_{m}^{2} \mathrm{e}^{i \Delta \beta z},
\end{array}
$$

where the subscript $s$ (resp. $p$ ) stands for signal (resp. pump). We introduce the superscript (1) for the parameters of the PPF. We will later use (2) for the SMF. For clarity, we drop the superscripts when defining the parameters in what follows. $z$ is the position along the fibre, $\tau=t-\beta_{1, s} z$ with $\beta_{1, s}=\left.[\mathrm{d} \beta(\omega) / \mathrm{d} \omega]\right|_{\omega_{0}}$ where $\beta(\omega)$ is the propagation constant, $\Delta \beta=2 \beta\left(\omega_{0}\right)-\beta\left(2 \omega_{0}\right)$ is the phase mismatch, $\Delta \beta_{1}=\beta_{1, s}-\beta_{1, p}$ is the temporal walkoff where $\beta_{1, p}=\left.[\mathrm{d} \beta(\omega) / \mathrm{d} \omega]\right|_{2 \omega_{0}} . \beta_{2, s}=\left.\left[\mathrm{d}^{2} \beta(\omega) / \mathrm{d} \omega^{2}\right]\right|_{\omega_{0}}$ and $\beta_{2, p}=\left.\left[\mathrm{d}^{2} \beta(\omega) / \mathrm{d} \omega^{2}\right]\right|_{2 \omega_{0}}$ are the group velocity dispersion coefficients. $\alpha_{s, p}$ are the loss coefficient and $\kappa$ is

\footnotetext{
*Electronic address: nicolas.englebert@ulb.ac.be
}

the second-order nonlinear parameter of the fibre. The third order nonlinearity is neglected because the PPF is much shorter than the total cavity length.

On the other hand, the signal evolution in the single-mode fibre (SMF) is described by the nonlinear Schrödinger equation (NLSE) [3]

$$
\frac{\partial A_{m}}{\partial z}=-\left(\frac{\alpha_{s}^{(2)}}{2}+i \frac{\beta_{2, s}^{(2)}}{2} \frac{\partial^{2}}{\partial \tau^{2}}-i \gamma\left|A_{m}\right|^{2}\right) A_{m}
$$

where $\gamma$ is the third-order nonlinear parameter of the fibre. The set of equations (S1)-(S5) constitute the full lumped model of the system. It is often referred to as a generalised Ikeda map [4]. This map can be reduced to a single mean-field equation following the approach described in $[1,2]$. We consider that $A_{m}$ remains constant over one roundtrip and integrate (S4) over the length of the PPF. We find

$$
B_{m} \approx B_{i n} \mathrm{e}^{-\frac{\alpha_{p}^{(1)} z}{2}}+\kappa \int_{-\infty}^{\infty} \mathcal{F}\left[A_{m}^{2}\right] \frac{\mathrm{e}^{i \Delta \beta z}-\mathrm{e}^{\hat{k} z}}{\Delta \beta+i \hat{k}} \mathrm{e}^{-i \Omega \tau} \mathrm{d} \Omega,
$$

where $\mathcal{F}[\cdot]$ stands for the Fourier transform operator and $\hat{k}=-\alpha_{p}^{(1)} / 2+i\left(\Delta \beta_{1} \Omega+\left(\beta_{2, p}^{(1)} \Omega^{2}\right) / 2\right)$. By substituting the latter expression in (S3) and integrating the result over the PPF length $L_{1}$ while keeping $A_{m}(z, \tau)$ constant, we find

$$
\begin{aligned}
A_{m}\left(L_{1}\right) \approx & A_{m}(0)-\left(\frac{\alpha_{s}^{(1)}}{2}+i \frac{\beta_{2, s}^{(1)}}{2} \frac{\partial^{2}}{\partial \tau^{2}}\right) L_{1} A_{m}(0) \\
& +i \kappa B_{i n} \mathrm{e}^{-i \xi} \frac{\sinh \xi}{\xi} L_{1} A_{m}^{*}(0) \\
& -\rho^{2}\left[A_{m}^{2} \otimes I(\tau)\right] A_{m}^{*}(0)
\end{aligned}
$$

where $\xi=L_{1}\left(\alpha_{p}^{(1)}+i \Delta \beta\right) / 2, \rho=\kappa L_{1}, I(\tau)=\mathcal{F}^{-1}[\hat{I}(\Omega)]$ is the kernel where $\mathcal{F}^{-1}[\cdot]$ stands for the inverse Fourier transform operator, $\hat{I}(\Omega)=\left(1-i x-\mathrm{e}^{-i x}\right) / x^{2}$ with $x(\Omega)=(\Delta \beta+i \hat{k}) L_{1}$. The NLSE (S5) can also be integrated over the SMF length $L_{2}$, from $L_{1}$ to $L_{1}+L_{2}=L$ 
while $A_{m}(z, \tau)$ is kept constant $[5,6]$. We find

$A_{m}(L) \approx A_{m}\left(L_{1}\right)-\left(\frac{\alpha_{s}^{(2)}}{2}+i \frac{\beta_{2, s}^{(2)}}{2} \frac{\partial^{2}}{\partial \tau^{2}}-i \gamma\left|A_{m}\right|^{2}\right) L_{1} A_{m}$.

We substitute (S7) in (S8) and keep only the first order terms, obtaining

$$
\begin{aligned}
A_{m}(L)- & A_{m}(0) \approx-\left(\frac{\alpha_{s}^{(1)}}{2}+i \frac{\beta_{2, s}^{(1)}}{2} \frac{\partial^{2}}{\partial \tau^{2}}\right) L_{1} A_{m}(0) \\
& +i \kappa B_{i n} \mathrm{e}^{-i \xi} \frac{\sinh \xi}{\xi} L_{1} A_{m}^{*}(0) \\
& -\rho^{2}\left[A_{m}^{2} \otimes I(\tau)\right] A_{m}^{*}(0) \\
& -\left(\frac{\alpha_{s}^{(2)}}{2}+i \frac{\beta_{2, s}^{(2)}}{2} \frac{\partial^{2}}{\partial \tau^{2}}-i \gamma\left|A_{m}(0)\right|^{2}\right) L_{2} A_{m}(0)
\end{aligned}
$$

At first order, under the hypothesis of high finesse and by introducing the detuning $\delta_{0}=2 k \pi-\varphi_{0}$ where $k$ is an integer, the boundary condition $(\mathrm{S} 1)$ can be written

$$
A_{m+1}(0) \approx\left(1-\frac{\mathcal{R}}{2}-i \delta_{0}\right) A_{m}(L),
$$

where $\mathcal{R}=1-\mathcal{T}$. By substituting ( $\mathrm{S} 9)$ in (S10), we find the mean-field equation

$$
\begin{aligned}
t_{R} \frac{\partial A}{\partial T} & =\left(-\frac{\Lambda}{2}-i \delta_{0}-i \frac{\beta_{2} L}{2} \frac{\partial^{2}}{\partial \tau^{2}}+i \gamma L_{2}|A|^{2}\right) A \\
& +\left(i \kappa B_{i n} L_{1} \mathrm{e}^{-\xi} \frac{\sinh \xi}{\xi}-\rho^{2}\left[A^{2} \otimes I(\tau)\right]\right) A^{*}
\end{aligned}
$$

where we have introduced the slow-time $T=n t_{R}$ with $t_{R}$ the rountripe time, $\Lambda=\alpha_{s}^{(1)} L_{1}+\alpha_{s}^{(2)} L_{2}+\mathcal{R}$ and $\underline{\beta_{2}} L=\beta_{2, s}^{(1)} L_{1}+\beta_{2, s}^{(2)} L_{2}$. Equation (S11) describes pattern formation in a singly resonant optical parametric oscillator with a Kerr section. It is a generalisation of the model introduced in [1]. Next, we further generalise the model to account for an intracavity amplifier at the signal frequency. Equation (S11) becomes [7]:

$$
\begin{aligned}
t_{R} \frac{\partial A}{\partial T} & =\left(-\frac{\alpha}{2}+\frac{g L_{3}}{2}-i \delta_{0}-i \frac{\beta_{2} L}{2} \frac{\partial^{2}}{\partial \tau^{2}}+i \gamma L_{2}|A|^{2}\right) A \\
& +\left(i \kappa B_{i n} L_{1} \mathrm{e}^{-\xi} \frac{\sinh \xi}{\xi}-\rho^{2}\left[A^{2} \otimes I(\tau)\right]\right) A^{*}
\end{aligned}
$$

where $g$ is the gain per meter and $L_{3}$ the amplifier length. As the erbium ions relaxation time $\left(\tau_{g}=10 \mathrm{~ms}\right)$ is much longer than the roundtrip time $\left(t_{R}=100 \mathrm{~ns}\right)$ and providing that $t_{R}\left\langle|A(T, \tau)|^{2}\right\rangle \ll \tau_{g} P_{\text {sat }}$, the gain evolution over one roundtrip is given by $[8,9]$

$$
t_{R} \frac{\partial g}{\partial T}=-t_{R}\left(\frac{1}{\tau_{g}}+\frac{\left\langle|u(T, \tau)|^{2}\right\rangle}{\tau_{g} P_{\text {sat }}}\right) g+\frac{t_{R} g_{0}}{\tau_{g}}
$$

where $\left\langle|u(T, \tau)|^{2}\right\rangle=\frac{1}{t_{R}} \int_{0}^{t_{R}}|u(T, \tau)|^{2} \mathrm{~d} \tau, g_{0}$ is the unsaturated gain and and $P_{\text {sat }}$ is the saturation power. This equation and (S12) describe the dynamics of our system. Because the gain dynamics is slow in our case, we make the approximation $\partial_{T} g=0$ which yields

$$
g=\frac{g_{0} L_{3} / 2}{1+\frac{\left\langle|u(T, \tau)|^{2}\right\rangle}{P_{\text {sat }}}} .
$$

In this paper, we focus on the detuning region $(0<$ $\left.\delta_{0}<0.3\right)$ where the intracavity filter does not impact the solitons. In that region, the average intracavity power $\left\langle|u(T, \tau)|^{2}\right\rangle$ is low and the gain stays close to its unsaturated value (i.e. $g \approx g_{0}$ ). The resonator intrinsic loss $\alpha$ can then be replaced by the effective loss $\Lambda_{e}$, defined as $[9]$

$$
\Lambda_{e}=\Lambda-g L_{3} \approx \Lambda-g_{0} L_{3}
$$

Moreover, the delayed third order term (describing upconversion) is also negligible in that region, leading to the following simplified mean-field equation, known as the parametrically driven nonlinear Schrödinger equation (PDNLSE) [10-12]

$$
\begin{aligned}
t_{R} \frac{\partial A}{\partial T}=\left(-\frac{\Lambda_{e}}{2}-i\right. & \left.\frac{\beta_{2} L}{2} \frac{\partial^{2}}{\partial \tau^{2}}-i \delta_{0}\right) A \\
& +\kappa B_{i n} L_{1} A^{*}+i \gamma L_{2}|A|^{2} A
\end{aligned}
$$

By introducing the parameters $T^{\prime}=\left(\Lambda_{e} T\right) / t_{R}, \Delta=$ $\delta_{0} / \Lambda_{e}, \tau^{\prime}=\sqrt{\frac{2 \Lambda_{e}}{\left|\underline{\beta_{2}} L\right|}}, u=\sqrt{\frac{\gamma L_{2}}{\Lambda_{e}}} A$ and $\mu=\kappa B_{i n} L_{1} / \Lambda_{e}$, we obtain the normalized, dimensionless version

$$
\frac{\partial u}{\partial T^{\prime}}=\left(-1+i\left(|u|^{2}-\Delta\right)+i \frac{\partial^{2}}{\partial \tau^{\prime 2}}\right) u+\mu u^{*} .
$$

\section{LINEAR STABILITY ANALYSIS}

To compute the linear stability of the homogeneous state solutions $u_{h}$ against generic perturbations $\xi(\tau, T)$, we first linearize the system around $u_{h}$ by introducing the ansatz $u(\tau, T)=u_{h}+\epsilon \xi(\tau, T)+c . c .(|\epsilon| \ll 1)$ in Eq. (S17). Keeping all the terms at first order in $\epsilon$, we obtain the linear equation

$$
\partial_{T}\left[\begin{array}{c}
\xi \\
\xi^{*}
\end{array}\right]=\left[\begin{array}{cc}
\mathrm{A} & \mathrm{B} \\
\mathrm{B}^{*} & \mathrm{~A}^{*}
\end{array}\right]\left[\begin{array}{c}
\xi \\
\xi^{*}
\end{array}\right]
$$

where

$$
\mathrm{A} \equiv-(1+i \Delta)+i \partial_{\tau}^{2}+2 i\left|u_{h}\right|^{2}, \quad \mathrm{~B} \equiv i u_{h}^{2}+\mu .
$$

To solve this equation, we consider modulated perturbation modes of the form $\xi(\tau, T)=a_{\Omega} e^{\sigma T+i \Omega \tau}+$ c.c., with $\Omega$ 
and $\sigma$ being the frequency and growth rate of the perturbation, respectively. This ansatz then leads to the linear system

$$
\left[\begin{array}{cc}
\mathrm{A}_{\Omega}-\sigma & \mathrm{B} \\
\mathrm{B}^{*} & \mathrm{~A}_{\Omega}^{*}-\sigma
\end{array}\right]\left[\begin{array}{l}
a_{\Omega} \\
a_{\Omega}^{*}
\end{array}\right]=\left[\begin{array}{l}
0 \\
0
\end{array}\right],
$$

with $\mathrm{A}_{\Omega} \equiv-(1+i \Delta)-i \Omega^{2}+2 i\left|u_{h}\right|^{2}$, which has a nontrivial solution if

$$
\sigma^{2}+2 \sigma+f(\Omega)=0, \quad f(\Omega)=\left|\mathrm{A}_{\Omega}\right|^{2}-|\mathrm{B}|^{2},
$$

is satisfied. This condition yields to the dispersion relation

$$
\sigma(\Omega)=-1+\sqrt{1-f(\Omega)},
$$

which relates the growth of the perturbation $\xi$ with its frequency. If $\operatorname{Re}[\sigma]$ is negative, the modulated perturbation $\xi$ decays, and $u_{h}$ is stable. However, when the contrary holds, the perturbation grows exponentially and $u_{h}$ is unstable. The transition occurs at the critical frequency $\Omega_{c}$ which satisfies simultaneously the conditions (i) $\left.\sigma(\Omega)\right|_{\Omega_{c}}=0$ and (ii) $\left.\sigma^{\prime}(\Omega)\right|_{\Omega_{c}}=0$, with (') denoting derivation with respect to $\Omega$.

\section{A. Stability of the trivial state}

For the trivial state $u_{h}=0, f(\Omega)=1-\mu^{2}+\left(\Delta+\Omega^{2}\right)^{2}$, and the conditions (i)-(ii) lead respectively to

$$
\mu^{2}=1+\left(\Delta+\Omega_{c}^{2}\right)^{2}, \quad\left(\Delta+\Omega_{c}^{2}\right) \Omega_{c}=0,
$$

which define the onset of the instability and the critical frequency $\Omega_{c}$ of the growing perturbation at the instability. Depending on $\Omega_{c}$, two different instabilities take place. For $\Omega_{c}=0$, a pitchfork bifurcation, occurs at $\mu=\mu_{p} \equiv \sqrt{1+\Delta^{2}}$, such that for $\mu<\mu_{p}, u_{h}=0$ is stable against homogeneous perturbations and unstable otherwise. This instability corresponds to the degenerate OPO threshold.

In contrast, when $\Omega_{c}=\Omega_{T} \equiv \sqrt{-\Delta}$ and $\Delta<0$, a Turing or modulation instability crops up at $\mu=\mu_{T} \equiv 1$, where $u_{h}=0$ becomes unstable against modulated perturbations of frequency $\Omega_{T}$. This instability corresponds to the non-degenerate OPO threshold.

\section{B. Stability of the non-trivial state}

The non-trivial homogeneous state can be written as $u_{h}=\sqrt{X} e^{i \phi}$, where $X$ satisfies $\mu^{2}=1+(X-\Delta)^{2}$, and $\cos (2 \phi)=\mu^{-1}$. For $\Delta>0$, two non-trivial states $u_{h}^{ \pm}$exist, corresponding to the intensities $X^{ \pm} \equiv \Delta \pm$ $\sqrt{\mu^{2}-1}$. However, for $\Delta<0$ the only nontrivial state corresponds to $u_{h}^{+}$(i.e., to $X^{+} \equiv \Delta+\sqrt{\mu^{2}-1}$ ).

In this case, $f=\left(2 X-\left(\Delta+\Omega^{2}\right)\right)^{2}-\Delta^{2}$, and the dispersion relation reads [13]

$$
\sigma^{ \pm}=-1+\sqrt{\Delta^{2}+1-\left(\Delta \pm 2 \sqrt{\mu^{2}-1}-\Omega^{2}\right)^{2}} .
$$

Here, the conditions (i)-(ii) become

$$
X_{c}^{ \pm}=\frac{1}{2}\left(\Omega_{c}^{2}+\Delta \pm \Delta\right), \quad\left(\Delta+\Omega_{c}^{2}-2 X\right) \Omega_{c}=0 .
$$

The condition $\Omega_{c}=0$ leads to two instabilities. The first one occurs at $(\mu, X)=\left(\mu_{p}, 0\right)$ and corresponds to the pitchfork bifurcation. For $\Delta<0, u_{h}^{+}$bifurcates from $\left(\mu, u_{h}\right)=\left(\mu_{p}, 0\right)$ towards $\mu>\mu_{p}$, and the pitchfork bifurcation is called supercritical. In contrast, for $\Delta>0$, $u_{h}^{-}$bifurcates towards $\mu<\mu_{p}$, and the pitchfork bifurcation is subcritical. The second instability occurs at $(\mu, X)=(1, \Delta)$ for $\Delta>0$, and corresponds to the saddlenode bifurcation $\mathrm{SN}_{h}$ where $u_{h}^{+}$and $u_{h}^{-}$meet one another (i.e., $X^{+}=X^{-}$).

For $\Omega_{c} \neq 0$, the conditions (i)-(ii) define the Turing instability, that in this case occurs at $\Delta=\Delta_{T} \equiv 0$ for a critical frequency $\Omega_{T}^{2}=2 \sqrt{\mu^{2}-1}$. Below and above this line, the non-trivial state $u_{h}$ is modulationally unstable.

For $\Delta<0, u_{h}^{+}$is modulationally unstable, and the most unstable perturbation frequency is $\Omega_{c}^{+}=$ $\sqrt{\Delta+2 \sqrt{\mu^{2}-1}}$, which leads to $\sigma_{\max }^{+}=-1+\sqrt{\Delta^{2}+1}$.

For $\Delta>0, u_{h}^{+}$preserves its modulationally unstable nature, and $u_{h}^{-}$is modulationally unstable for $1<\mu<\sqrt{\Delta^{2} / 4+1}$, with a most unstable frequency $\Omega_{c}^{-}=\sqrt{\Delta-2 \sqrt{\mu^{2}-1}}$. In contrast, when $\sqrt{\Delta^{2} / 4+1}<$ $\mu<\mu_{p}$, the most unstable mode corresponds to $\Omega=0$, therefore $u_{h}^{-}$is unstable to homogeneous perturbations.

\section{PHASE MODULATION}

Cavity solitons (CS) are known to be attracted to phase maxima of the driving beam [14]. In our experiment, we use a similar technique to lock the parametric cavity solitons (PCSs) on a $4.6 \mathrm{GHz}$ grid. We imprint a periodic phase modulation $\phi(\tau)$ onto the driving laser prior to its frequency doubling. The resulting beam is phase modulated at twice the initial modulation frequency (see Fig. 3). Writing $\mu(\tau)=\mu_{0} e^{i 2 \phi(\tau)}$, the meanfield equation (S17) becomes

$$
\frac{\partial u}{\partial T}=\left(-1+i\left(|u|^{2}-\Delta\right)+i \frac{\partial^{2}}{\partial \tau^{2}}\right) u+\mu_{0} e^{i 2 \phi(\tau)} u^{*}
$$

By substituting $u=\bar{u} e^{i \phi(\tau)}$ into equation (S26), we find

$$
\begin{array}{r}
\frac{\partial \bar{u}}{\partial T}=\left(-\left(1+\phi^{\prime \prime}\right)+i\left(|\bar{u}|^{2}-\left(\Delta+\phi^{2}\right)\right)+i \frac{\partial^{2}}{\partial \tau^{2}}-\right. \\
\left.-2 \phi^{\prime} \frac{\partial}{\partial \tau}\right) \bar{u}+\mu_{0} \bar{u}^{*},
\end{array}
$$

where $\phi^{\prime}=\frac{d \phi}{d \tau}$ and $\phi^{\prime \prime}=\frac{d^{2} \phi}{d \tau^{2}}$. The impact of $\phi^{\prime \prime}$ (resp. $\phi^{\prime 2}$ ) over the total losses (resp. detuning) is small [14]. The term $2 \phi^{\prime} \partial_{\tau}$ provides a local $\tau$-dependent variation of 
the group velocity. When $\phi^{\prime}>0$ (resp. $\left.\phi^{\prime}<0\right)$, the soliton suffers a delay (resp. advancement) with respect to the carrier frequency reference frame. This implies that individual PCS will lock to a phase modulation maximum.

\section{References}

1. Mosca, S. et al. Modulation Instability Induced Frequency Comb Generation in a Continuously Pumped Optical Parametric Oscillator. Physical Review Letters 121, 093903 (2018).

2. Leo, F. et al. Walk-Off-Induced Modulation Instability, Temporal Pattern Formation, and Frequency Comb Generation in Cavity-Enhanced Second-Harmonic Generation. Physical Review Letters 116, 033901 (2016).

3. Agrawal, G. Nonlinear Fiber Optics (Academic Press, 2013).

4. Ikeda, K. Multiple-valued stationary state and its instability of the transmitted light by a ring cavity system. Optics Communications 30, 257-261 (1979).

5. Lugiato, L. A. \& Lefever, R. Spatial Dissipative Structures in Passive Optical Systems. Physical Review Letters 58, 2209-2211 (1987).

6. Haelterman, M., Trillo, S. \& Wabnitz, S. Dissipative modulation instability in a nonlinear dispersive ring cavity. Optics Communications 91, 401-407 (1992).
7. Komarov, A., Leblond, H. \& Sanchez, F. Multistability and hysteresis phenomena in passively mode-locked fiber lasers. Physical Review A 71, 053809 (2005).

8. Haboucha, A., Leblond, H., Salhi, M., Komarov, A. \& Sanchez, F. Analysis of soliton pattern formation in passively mode-locked fiber lasers. Physical Review A 78, 043806 (2008).

9. Englebert, N., Gorza, S.-P. \& Leo, F. Temporal Solitons in a Coherently Driven Active Resonator. arXiv:200\%.15630 [physics] (2020).

10. Miles, J. W. Parametrically excited solitary waves. fr. Journal of Fluid Mechanics 148, 451-460 (1984).

11. Denardo, B. et al. Observations of localized structures in nonlinear lattices: Domain walls and kinks. Physical Review Letters 68, 1730-1733 (1992).

12. Longhi, S. Ultrashort-pulse generation in degenerate optical parametric oscillators. Optics Letters 20, 695-697 (1995).

13. Longhi, S. \& Geraci, A. Modulational instability oscillation and solitary waves in a nonlinear dispersive cavity with parametric gain. Applied Physics Letters 67, 30603062 (1995).

14. Jang, J. K., Erkintalo, M., Coen, S. \& Murdoch, S. G. Temporal tweezing of light through the trapping and manipulation of temporal cavity solitons. Nature Communications 6, 7370 (2015). 
a

$\Delta$

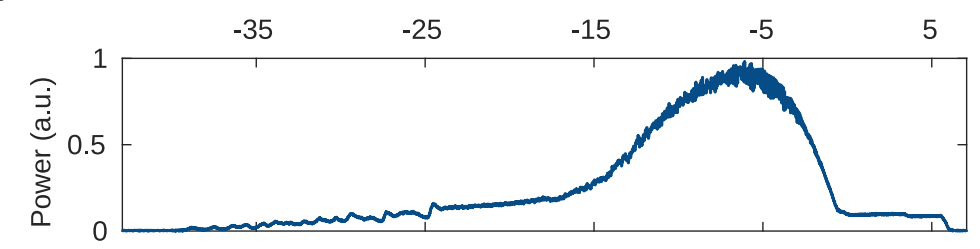

b

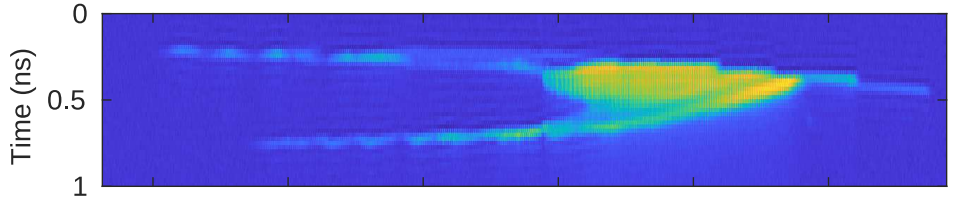

c

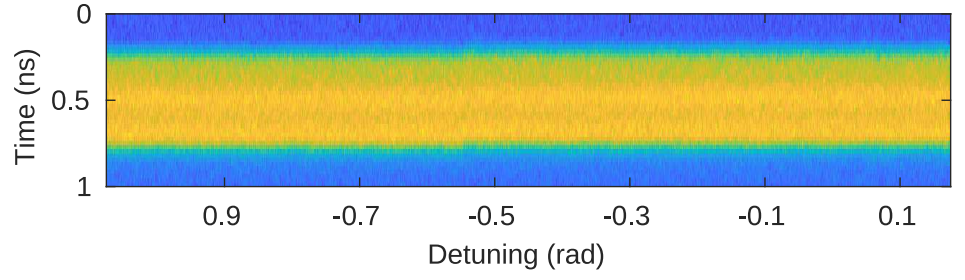

d
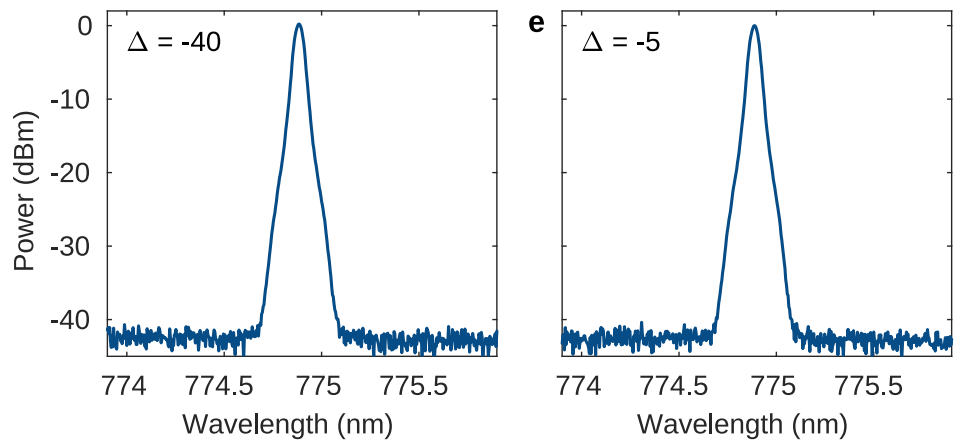

Fig. S1. Spontaneous Parametric Cavity Solitons generation. a, Cavity resonance measured with a $200 \mathrm{kHz}$ photodiode. The scan leads to the generation of a multiple soliton-step, i.e. the spontaneous generation of multiple PCSs. b, Similar scan performed with a $12 \mathrm{GHz}$ detection system. The oscillation is initiated on the edges of the driving pulse, after which the signal broadens to reach the same duration as the pump pulse and eventually collapses on the soliton state. c, Corresponding pump pulse profile. It remains unchanged throughout the scan which validates the constant pump approximation used in our model. $\mathbf{d}$, Pump spectrum below and above (e) the oscillation threshold. The absence of spectral broadening further confirms that cascaded nonlinearites do not play a significant role in our system. 